KUNS-1700

\title{
Can we distinguish SUSY breaking terms between weakly and strongly coupled heterotic string theories?
}

\author{
Tatsuo Kobayashi卂 \\ Department of Physics, Kyoto University Kyoto 606-8502, Japan
}

\begin{abstract}
We study soft SUSY breaking terms in heterotic M-theory. We show that both weakly and strongly coupled heterotic string models lead to the same relations of soft SUSY breaking terms, $A=-M$ and $m^{2}=M^{2} / 3$, up to $O\left((\alpha T / S)^{2}\right)$.
\end{abstract}

\footnotetext{
*E-mail address: kobayash@gauge.scphys.kyoto-u.ac.jp
} 


\section{Introduction}

Supersymmetry (SUSY) breaking is important to connect superstring theory with our real world. Under the assumption that the dilation field $S$ and moduli fields $T^{i}$ contribute to SUSY breaking, soft SUSY breaking terms have been studied within the framework of effective supergravity theory inspired by superstring theory, in particular weakly coupled heterotic string theory [1]-[5]. Their phenomenological implications have been investigated from various viewpoints. The weakly coupled heterotic string theory leads to the following relations among soft SUSY breaking terms,

$$
\begin{gathered}
h^{i j k}=-M \hat{Y}^{i j k}, \\
m_{i}^{2}+m_{j}^{2}+m_{k}^{2}=|M|^{2},
\end{gathered}
$$

where $M$ is the gaugino mass, $h^{i j k}$ is the trilinear coupling of scalar fields corresponding to

the Yukawa coupling $\hat{Y}^{i j k}$ and $m_{i}$ is the soft scalar mass of scalar field $\phi_{i}$ for non-vanishing Yukawa couplings $\hat{Y}^{i j k}$ if Yukawa couplings in the supergravity basis are field-independent, the scalar potential is $T$-duality invariant and the vacuum energy vanishes. In particular, the case with universal Kähler metric of chiral matter fields, e.g. orbifold models with only untwisted sectors and the large $T$ limit of Calabi-Yau models, leads to the universal scalar mass, $m_{0}$,

$$
m_{0}^{2}=\frac{1}{3}|M|^{2} .
$$

These relations (1) and (2) are one-loop renormalization group ( $R G$ ) invariant if the Yukawa coupling is on the RG-invariant trajectory $\hat{Y}^{i j k}=g C^{i j k}$, where $g$ is the gauge coupling and $C^{i j k}$ is a constant determined by group-theoretical factors [6, 7]. T-duality anomaly induces a certain correction to the relations. On the other hand, the two-loop and all order RG-invariant relations have been derived in Refs. [8, 9], and those are exactly coincide with the relations induced by the $T$-duality anomaly for finite theories. These results on the soft SUSY breaking terms might be a remnant of finiteness of string theory.

Similarly, effective supergravity theory inspired by strongly coupled heterotic theory, M-theory on $S^{1} / Z_{2}$ [10], has been obtained [11]-20]. That is different from the weakly coupled one by terms including the factor $\alpha T / S$. In addition, soft SUSY breaking terms have been discussed with use of this effective supergravity theory [21]-28]. The results

\footnotetext{
${ }^{\dagger}$ For phenomenological aspects, see e.g. Ref. 229].
} 
seem to differ from those of weakly coupled theory by the factor, $\alpha T / S$. Thus, in the literature it has been deduced that the heterotic M-theory would lead to phenomenological aspects different from the weakly coupled one.

In this paper we study soft SUSY breaking terms derived from heterotic M-theory and we will show heterotic M-theory also leads to the relations (10), (3) up to $O\left((\alpha T / S)^{2}\right)$. Thus, at this level we can not distinguish soft SUSY breaking terms between weakly and strongly coupled heterotic string models.

\section{$2 \quad$ Weakly coupled heterotic models}

At first we give a brief review on soft SUSY breaking terms derived from generic supergravity theory. We start with the following Kähler potential $K$ and the superpotential $W$

$$
\begin{aligned}
K & =\tilde{K}\left(\Phi_{a}, \bar{\Phi}^{a}\right)+K_{i}^{i}\left(\Phi_{a}, \bar{\Phi}^{a}\right)\left|\Phi_{i}\right|^{2}, \\
W & =\tilde{W}\left(\Phi_{a}\right)+\frac{1}{6} Y^{i j k} \Phi_{i} \Phi_{j} \Phi_{k},
\end{aligned}
$$

where chiral superfields have been classified into two types: $\Phi_{a}$ would have large vacuum expectation values and the other light fields are denoted by $\Phi_{i}$. All of them are denoted by $\Phi_{I}$. Here $\tilde{W}\left(\Phi_{a}\right)$ is a nonperturbative superpotential leading to SUSY breaking. We have neglected supersymmetric mass terms of $\Phi_{i}$. The scalar potential is obtained as

$$
V=F_{I} F^{J} K_{J}^{I}-3 e^{G}
$$

where $F_{I}$ are $F$-terms of $\Phi_{I}$ and $G=K+\ln |W|^{2}$. Here we assume that the fields $\Phi_{a}$ develop their vacuum expectation values and their $F$-terms contribute to SUSY breaking. Then, taking the flat limit, we can expand the scalar potential around nonvanishing $\Phi_{a}$ and $F_{a}$,

$$
V=V_{0}+\sum_{i}\left|\frac{\partial W_{e f f}}{\partial \phi_{i}}\right|^{2}+\frac{1}{2} m_{i}^{2}\left|\phi_{i}\right|^{2} K_{i}^{i}+\left(\frac{1}{6} h^{i j k} \phi_{i} \phi_{j} \phi_{k} \prod_{\ell=i, j, k}\left(K_{\ell}^{\ell}\right)^{1 / 2}+h . c .\right)+\cdots,
$$

where $\phi_{i}$ is the scalar component of $\Phi_{i}$, and the first term of RHS is the vacuum energy obtained as $V_{0}=F_{a} F^{b} K_{b}^{a}-3 m_{3 / 2}^{2}$ with $m_{3 / 2}^{2} \equiv e^{G}$. The second term of RHS is globally 
supersymmetric terms where the Yukawa coupling $\hat{Y}^{i j k}$ in the global SUSY basis is obtained for the canonical field,

$$
\left|\hat{Y}^{i j k}\right|^{2}=e^{\tilde{K}}\left|Y^{i j k}\right|^{2}\left(K_{i}^{i} K_{j}^{j} K_{k}^{k}\right)^{-1} .
$$

The other terms correspond to SUSY breaking terms, that is, the soft scalar mass $m_{i}$ and the trilinear coupling $h^{i j k}$ are obtained [30, 2]

$$
\begin{aligned}
m_{i}^{2} & =m_{3 / 2}^{2}-F_{a} F^{b} \partial^{a} \partial_{b}\left(\ln K_{i}^{i}\right)+V_{0}, \\
h^{i j k} & =\hat{Y}^{i j k} F_{a} \partial^{a}\left(\tilde{K}-\ln \left(K_{i}^{i} K_{j}^{j} K_{k}^{k}\right)\right)+\cdots,
\end{aligned}
$$

where $\partial^{a}\left(\partial_{b}\right)$ denotes $\partial / \partial \Phi_{a}$. Here the ellipsis denotes the term including derivatives of $Y^{i j k}$ by the fields $\Phi_{a}$. Hereafter we restrict ourselves to the field-independent Yukawa couplings $Y^{i j k}$. The other SUSY breaking terms in the scalar potential are suppressed by the Planck scale. The gaugino mass $M_{\alpha}$ is obtained from the gauge kinetic function $f\left(\Phi_{a}\right)_{\alpha}$

$$
M_{\alpha}=F_{a} \partial^{a}\left(\ln R e f_{\alpha}\right)
$$

and the gauge coupling $g$ is obtained

$$
1 / g_{\alpha}^{2}=\operatorname{Re}\left(f_{\alpha}\right)
$$

The weakly coupled heterotic string model leads to the following gauge kinetic function and the Kähler potential,

$$
\begin{aligned}
f_{\alpha} & =S, \\
K & =-\ln (S+\bar{S})-3 \ln (T+\bar{T})+3(T+\bar{T})^{n_{i}}\left|\Phi_{i}\right|^{2},
\end{aligned}
$$

where $S$ and $T$ are the dilaton field and the overall moduli field, and $n_{i}$ are modular weights of $\Phi_{i}$.

Here we restrict ourselves to the case with the universal Kähler metric $n_{i}=-1$, which correspond to the orbifold models with only untwisted matter fields and the large $T$ limit of Calabi-Yau models. Because only the strongly coupled heterotic model with the universal Kähler metric has been known and it is not clear that we can have twisted sectors in $N=1$ strongly coupled models.

\footnotetext{
${ }^{\ddagger}$ In general, the level of gauge group $k_{\alpha}$ is not equal to unity, but here we restrict ourselves to the case with $k_{\alpha}=1$.
} 
Here we assume that $F$-terms of $S$ and $T$ contribute SUSY breaking with vanishing vacuum energy, $V_{0}=0$. Following Ref. [3], we parameterize the $F$-terms by the goldstino angle $\theta$,

$$
\begin{aligned}
& F^{S}=\sqrt{3} m_{3 / 2}(S+\bar{S}) \sin \theta e^{-i \gamma_{S}} \\
& F^{T}=m_{3 / 2}(T+\bar{T}) \cos \theta e^{-i \gamma_{T}}
\end{aligned}
$$

where $\gamma_{S}$ and $\gamma_{T}$ are phases of $F^{S}$ and $F^{T}$. Hereafter we neglect CP phases $\gamma_{S}$ and $\gamma_{T}$ for simplicity. Then we have

$$
\begin{aligned}
M & =\sqrt{3} m_{3 / 2} \sin \theta, \\
m_{i}^{2} & =m_{3 / 2}^{2} \sin ^{2} \theta, \\
h^{i j k} & =-\sqrt{3} \hat{Y}^{i j k} m_{3 / 2} \sin \theta .
\end{aligned}
$$

Thus, the relations (11) and (3) hold in this case. These relations are obtained even if we take into account the CP phases, $\gamma_{S}$ and $\gamma_{T}$. Furthermore, we have the linear relation between the gauge coupling $g$ and the Yukawa coupling,

$$
\left|\hat{Y}^{i j k}\right|^{2}=g^{2}\left|Y^{i j k}\right|^{2} / 27
$$

Note that the gauge coupling is obtained $1 / g^{2}=R e S$.

Here we give a comment on the case with many modular weights $n_{i}$. Let us consider T-duality transformation,

$$
\begin{aligned}
T & \rightarrow(a T-i b) /(i c T+d) \\
\Phi_{i} & \rightarrow(i c T+d)^{n_{i}} \Phi_{i}
\end{aligned}
$$

where $a, b, c$ and $d$ are integers satisfying $a d-b c=1$. Here we require $G$ as well as the potential $V$ should be invariant under the above duality transformation. That implies that the superpotential $W$ should transform $W \rightarrow(i c T+d)^{-3} W$. Therefore, the Yukawa couplings are allowed for the fields whose modular weights satisfy $n_{i}+n_{j}+n_{k}=-3$. In this case, we have the relations (1),(2). Also, in this case, we have the linear relation between the gauge and Yukawa couplings (19). These results are extended to the case with three diagonal moduli fields [4, 5]. 


\section{$3 \quad$ Heterotic M-theory}

Strongly coupled $E_{8} \times E_{8}$ heterotic string theory has been considered as M-theory compactified on $S^{1} / Z_{2}$. Its effective supergravity theory has been obtained at order of $\kappa^{4 / 3}$. That is, in the strongly coupled case, $\alpha T / S$ is sizable, where $\alpha$ is written by the anomaly, and the gauge kinetic functions and Kähler potential have correction terms. The corrections have been known up to $O\left((\alpha T / S)^{2}\right)$,

$$
\begin{aligned}
f_{o} & =S(1+\alpha T / S), \quad f_{h}=S(1-\alpha T / S) \\
K & =-\ln (S+\bar{S})-3 \ln (T+\bar{T}) \\
& +\frac{3}{T+\bar{T}}\left[1+\frac{\alpha(T+\bar{T})}{3(S+\bar{S})}\right]\left|\Phi_{o}\right|^{2}+\frac{3}{T+\bar{T}}\left[1-\frac{\alpha(T+\bar{T})}{3(S+\bar{S})}\right]\left|\Phi_{h}\right|^{2},
\end{aligned}
$$

where $f_{o}$ and $f_{h}$ are the gauge kinetic functions of the observable and hidden sectors, respectively and similarly $\Phi_{o}$ and $\Phi_{h}$ denote chiral superfields of the untwisted observable and hidden sectors, which correspond to $n_{i}=-1$ in eq.(14) for the weakly coupled case. It is remarkable that the Kähler potential is known up to $O\left((\alpha T / S)^{2}\right)$ and thus any calculation is reliable up to $O\left((\alpha T / S)^{2}\right)$. Only this viewpoint is different from previous calculations of SUSY breaking terms in heterotic M-theory.

Here we assume the following superpotential,

$$
W=\tilde{W}(S, T)+\frac{1}{6} Y_{o}^{i j k} \Phi_{o i} \Phi_{o j} \Phi_{o k}+\frac{1}{6} Y_{h}^{i j k} \Phi_{h i} \Phi_{h j} \Phi_{h k},
$$

where $\tilde{W}(S, T)$ is a nonperturbative superpotential of $S$ and $T$. We restrict ourselves to the case that the Yukawa couplings $Y_{o}^{i j k}$ and $Y_{h}^{i j k}$ are field-independent.

Now let us assume that $F$-terms of $S$ and $T$ contribute SUSY breaking and the vacuum energy $V_{0}$ vanish. We can use the same parameterization of $F$-terms as eq.(15) because the Kähler metric of $S$ and $T$ is same. We use the formulae eqs.(9), (10) and (11) for eqs.(22) and (23). Then we obtain the gaugino mass $M_{o}$, the trilinear coupling $h_{o}^{i j k}$ and the soft scalar mass $m_{o}$ in the observable sector,

$$
\begin{aligned}
M_{o}= & \frac{\sqrt{3} m_{3 / 2}}{(S+\bar{S})+\alpha(T+\bar{T})}\left((S+\bar{S}) \sin \theta+\frac{\alpha(T+\bar{T})}{\sqrt{3}} \cos \theta\right) \\
m_{o}^{2}= & m_{3 / 2}^{2}-\frac{3 m_{3 / 2}^{2}}{3(S+\bar{S})+\alpha(T+\bar{T})} \\
& \times\left\{\alpha(T+\bar{T})\left(2-\frac{\alpha(T+\bar{T})}{3(S+\bar{S})+\alpha(T+\bar{T})}\right) \sin ^{2} \theta\right.
\end{aligned}
$$




$$
\begin{aligned}
+ & (S+\bar{S})\left(2-\frac{3(S+\bar{S})}{3(S+\bar{S})+\alpha(T+\bar{T})}\right) \cos ^{2} \theta \\
& \left.-\frac{2 \sqrt{3} \alpha(T+\bar{T})(S+\bar{S})}{3(S+\bar{S})+\alpha(T+\bar{T})} \sin \theta \cos \theta\right\}, \\
h_{o}^{i j k}= & \sqrt{3} m_{3 / 2} \hat{Y}_{o}^{i j k}\left\{\left(-1+\frac{3 \alpha(T+\bar{T})}{3(S+\bar{S})+\alpha(T+\bar{T})}\right) \sin \theta\right. \\
+ & \left.\sqrt{3}\left(-1+\frac{3(S+\bar{S})}{3(S+\bar{S})+\alpha(T+\bar{T})}\right) \cos \theta\right\} .
\end{aligned}
$$

The same soft SUSY breaking terms are obtained for the hidden sector except replacing $\alpha$ by $-\alpha$.

Since we know the Kähler potential up to $O\left((\alpha S / T)^{2}\right)$, we compare these soft SUSY breaking terms up to $O\left((\alpha S / T)^{2}\right)$, and then find

$$
\begin{aligned}
h_{o}^{i j k} & =-\hat{Y}_{o}^{i j k} M_{o}+O\left((\alpha S / T)^{2}\right), \\
m_{o}^{2} & =\frac{1}{3} M_{o}^{2}+O\left((\alpha S / T)^{2}\right) .
\end{aligned}
$$

Note that in these relations there is no correction term linear to $\alpha T / S$. The same relations are obtained for the hidden sector, although the gaugino mass in the hidden sector $M_{h}$ is different from $M_{o}$. If there is a matter field charged under both observable and hidden gauge groups (like a twisted matter field in orbifold models), one could see the difference between $M_{o}$ and $M_{h}$. Similar calculations have been done in Ref. 228], where the square root of eq.(26) was taken and it was deduced that the relation (2) for the soft scalar masses have a correction of $O(\alpha S / T)$. However, the soft mass squared $m^{2}$ is much more essential than the soft mass $m$ itself from the viewpoint that the soft mass squared $m^{2}$ itself is derived from the scalar potential (]). Thus, it is more significant to compare $m^{2}$ than $m$.

We have neglected the CP phases $\gamma_{S}$ and $\gamma_{T}$. However, we obtain the same results (28) and (29) even if we take into account the CP phases. Then, here we conclude that the relations (1) and (3) still hold for heterotic M-theory up to $O\left((\alpha S / T)^{2}\right)$. Thus, we can not distinguish soft SUSY breaking terms between weakly and strongly coupled heterotic string models. Of course, the ratio of the gaugino mass to the gravitino mass is different between them, but that includes the goldstino angle, which is a free parameter. Also, as said above, the gaugino masses $M_{o}$ and $M_{h}$ in the observable and hidden sectors are different from each other, but this difference does not appear, because the observable and hidden sectors are completely decoupled and there is no mixing like a twisted sector of orbifold models. 
Furthermore, the linear relations between the gauge couplings and Yukawa couplings still hold,

$$
\left(\hat{Y}_{o}^{i j k}\right)^{2} \propto g_{o}^{2}, \quad\left(\hat{Y}_{h}^{i j k}\right)^{2} \propto g_{h}^{2} .
$$

Note that the gauge couplings of the observable and hidden sectors are obtained $1 / g_{o}^{2}=$ $\operatorname{Re}(S+\alpha T)$ and $1 / g_{h}^{2}=\operatorname{Re}(S-\alpha T)$.

All of the results, the relations of soft SUSY breaking terms and the linear relations between the gauge and Yukawa couplings would be understood if we write the Kähler metric $K_{o i}^{i}$ of the observable chiral field $\Phi_{o}$ as follows,

$$
K_{o i}^{i}=\frac{3}{T+\bar{T}}[1+\alpha(T+\bar{T}) /(S+\bar{S})]^{1 / 3}+O\left((\alpha T / S)^{2}\right) .
$$

We need to know higher order corrections of Kähler metric to distinguish the weakly and strongly coupled heterotic string models. If the Kähler metric $K_{o i}^{i}$ of the observable chiral field $\Phi_{o}$ is obtained

$$
K_{o i}^{i}=\frac{3}{T+\bar{T}}[1+\alpha(T+\bar{T}) /(S+\bar{S})]^{1 / 3}+O\left((\alpha T / S)^{n}\right),
$$

we would have the relations (11) and (3) up to $O\left((\alpha T / S)^{n}\right)$ and could not distinguish at this order. In fact, this belongs to the class of supergravity models leading to the RG-invariant relations, which have been discussed in Ref. [7].

Of course, the presence of 5-branes is non-trivial in heterotic M-theory. Thus, if Fterms of the 5-brane moduli fields contribute to SUSY breaking, that gives a certain type of difference from the weakly coupled heterotic string models as discussed in Ref. 31. 35. (However, with suppressed F-terms of 5-brane moduli fields, we can not see the difference.)

Here we give a comment on $\sigma$-model corrections. Including the $\sigma$-models corrections, the term $-3 \ln (T+\bar{T})$ of Kähler potential in eqs.(14) and (23) is shifted [36],

$$
-3 \ln (T+\bar{T}) \rightarrow-3 \ln (T+\bar{T})+\frac{A_{03}}{(T+\bar{T})^{3}},
$$

and the Kähler metric of $\Phi$ is corrected

$$
\frac{3}{(T+\bar{T})}|\Phi|^{2} \rightarrow \frac{3}{(T+\bar{T})}\left(1+\frac{B_{03}}{(T+\bar{T})^{3}}\right)|\Phi|^{2},
$$

for the weakly coupled model and

$$
\frac{3}{(T+\bar{T})}\left(1+\frac{\alpha(T+\bar{T})}{3(S+\bar{S})}\right)\left|\Phi_{o}\right|^{2} \rightarrow \frac{3}{(T+\bar{T})}\left(1+\frac{\alpha(T+\bar{T})}{3(S+\bar{S})}+\frac{B_{03}}{(T+\bar{T})^{3}}\right)\left|\Phi_{o}\right|^{2},
$$


for the observable sector of the strongly coupled model, where the coefficients $A_{03}$ and $B_{03}$ are of $O(1)$ and depend on geometrical aspects of the Calabi-Yau manifold [37, 25]. In the orbifold case, the coefficients $A_{03}$ and $B_{03}$ vanish and there is no such correction. While the parameterization of $F^{S}(15)$ holds true, the parameterization of $F^{T}$ changes,

$$
F^{T}=m_{3 / 2}(T+\bar{T})\left(1-\frac{2 A_{03}}{(T+\bar{T})^{3}}\right) \cos \theta .
$$

In both of weakly and strongly coupled models, we have

$$
\begin{aligned}
h^{i j k} & =-\hat{Y}^{i j k} M\left(1+\sqrt{3} \frac{A_{03}-3 B_{03}}{(T+\bar{T})^{3}} \cot \theta\right), \\
m^{2} & =\frac{1}{3} M^{2}\left(1+\frac{4\left(A_{03}-3 B_{03}\right)}{(T+\bar{T})^{3}} \cot ^{2} \theta\right),
\end{aligned}
$$

up to $O\left(1 /(T+\bar{T})^{6}\right)$ and $O\left((\alpha T / S)^{2}\right)$. For the model with $A_{03}=3 B_{03}$, such corrections vanish, but for other models, this type of corrections lead to significant difference between different weakly coupled Calabi-Yau models. However, such correction becomes less important in the strongly coupled model, which corresponds to $1 /(T+\bar{T})^{3}<<1$. the strongly coupled model we have rather the universal relations (11) and (3) for different values of $A_{03}$ and $B_{03}$. The deviation from (11), (3) would imply effects due to $A_{03}$ and $B_{03}$ rather in weakly coupled models.

\section{Conclusions}

We have studied soft SUSY breaking terms in the strongly coupled heterotic string model.

We have found that weakly and strongly coupled models lead to the same relations (1), (3) up to $O\left((\alpha T / S)^{2}\right)$. Thus we can not distinguish them at the present knowledge and we need to know higher order of the Kähler potential, but such calculations are difficult.

\section{Acknowledgment}

The author would like to thank K. Choi, Y. Kawamura and A. Lukas for useful discussions. He also thanks the Summer Institute 2000, Yamanashi, Japan.

\footnotetext{
$\S_{\text {See also Ref. } 25 .}$
} 


\section{References}

[1] L.E. Ibáñez and D. Lüst, Nucl. Phys. B382 (1992) 305;

B. de.Carlos, J.A. Casas and C. Muñoz, Phys. Lett. B299 (1993) 234.

[2] V.S. Kaplunovsky and J. Louis, Phys. Lett. B306 (1993) 269.

[3] A. Brignole, L.E. Ibáñez and C. Muñoz, Nucl. Phys. B422 (1994) 125.

[4] T. Kobayashi, D. Suematsu, K. Yamada and Y. Yamagishi, Phys. Lett. B348 (1995) 402.

[5] A. Brignole, L. E. Ibáñez, C. Muñoz and C. Scheich, Z. Phys. C74 (1997) 157.

[6] D.R.T. Jones, L. Mezincescu and Y.P. Yao, Phys. Lett. B148 (1984) 317;

I. Jack and D.R.T. Jones, Phys. Lett. B349 (1995) 294;

D.I. Kazakov, M.Yu. Kalmykov, I.N. Kondrashuk and A.V. Gladyshev, Nucl. Phys. B471 (1996) 387;

I. Jack, D.R.T. Jones and A. Pickering, Phys. Lett. B426 (1998) 73.

[7] Y. Kawamura, T. Kobayashi and J. Kubo, Phys. Lett. B405 (1997) 64.

[8] T. Kobayashi, J. Kubo, M. Mondragón and G. Zoupanos, Nucl. Phys. B511 (1998) 45.

[9] T. Kobayashi, J. Kubo and G. Zoupanos, Phys. Lett. B427 (1998) 291.

[10] P. Hořava and E. Witten, Nucl. Phys. B460 (1996) 506; Nucl.Phys.B475,(1996) 94;

P. Hořava, Phys. Rev. D54 (1996) 7561;

E. Witten, Nucl. Phys. B471 (1996) 135.

[11] T. Banks and M. Dine, Nucl. Phys. B479 (1996) 173.

[12] I. Antoniadis and M. Quirós, Phys. Lett. B392 (1997) 61; Phys. Lett. B416 (1998) 327.

[13] H.P. Nilles and S. Stieberger, Nucl. Phys. B499 (1997) 3.

[14] T. Li, J.L. Lopez and D.V. Nanopoulos, Phys. Rev. D56 (1997) 2602. 
[15] E. Dudas and C. Grojean, Nucl. Phys. B507 (1997) 553.

[16] K. Choi, Phys. Rev. D56 (1997) 6588.

[17] A. Lukas, B.A. Ovrut and D. Waldram, Nucl. Phys. B532 (1998) 43.

[18] A. Lukas, B.A. Ovrut, K.S. Stelle and D. Waldram, Nucl. Phys. B552 (1999) 246.

[19] J. Ellis, Z. Lalak, S. Pokorski and W. Pokorski, Nucl. Phys. B540 (1998) 149.

[20] J. Ellis, Z. Lalak and W. Pokorski, Nucl. Phys. B559 (1999) 71.

[21] H.P. Nilles, M. Olechowski and M. Yamaguchi, Phys. Lett. B415 (1997) 24; Nucl. Phys. B530 (1998) 43.

[22] Z. Lalak and S. Thomas, Nucl. Phys. B515 (1998) 55.

[23] E. Dudas, Phys. Lett. B416 (1998) 309.

[24] A. Lukas, B.A. Ovrut and D. Waldram, Phys. Rev. D57 (1998) 7529.

[25] K. Choi, H.B. Kim and C. Muñoz, Phys. Rev. D57 (1998) 7521.

[26] T. Li, Phys. Rev. D57 (1998) 7539.

[27] J. Ellis, Z. Lalak, S. Pokorski and S. Thomas, Nucl. Phys. B563 (1999) 107.

[28] T. Li, Nucl. Phys. B564 (2000) 441.

[29] D. Bailin,G.V. Kraniotis and A. Love, Nucl. Phys. B556 (1999) 23;

S.A. Abel and C.A. Savoy, Phys. Lett. B444 (1998) 119;

J.A. Casas, A. Ibarra and C. Muñoz, ; Nucl. Phys. B554 (1999) 67;

C.S. Huang, T. Li, W. Liao and Q.S. Yan and S.H. Zhu, hep-ph/9810412;

T. Li, hep-ph/9903371;

S. Khalil, G. Lazarides and C. Pallis, hep-ph/0005021.

[30] S. K. Soni and H. A. Weldon, Phys. Lett. B126 (1983) 215.

[31] A. Lukas, B.A. Ovrut and D. Waldram, Phys. Rev. D59 (1999) 106005;

R. Donagi, A. Lukas, B.A. Ovrut and D. Waldram, JHEP 9905 (1999) 018; JHEP 9906 (1999) 034. 
[32] A. Lukas, B.A. Ovrut and D. Waldram, JHEP 9904 (1999) 009.

[33] T. Kobayashi, J. Kubo and H. Shimabukuro, Nucl. Phys. B580 (2000) 3.

[34] D.G. Cerdeno and C. Munoz, Phys. Rev. D61 (2000) 016001.

[35] D. Bailin,G.V. Kraniotis and A. Love, Phys. Lett. B463 (1999) 174;

Phys. Lett. B483 (2000) 425.

[36] K. Choi, Z. Phys. C39 (1988) 219.

[37] A. Klemm and S. Theisen, Nucl. Phys. B389 (1993) 153;

A. Font, Nucl. Phys. B391 (1993) 153;

H.B. Kim and C. Munoz, Z. Phys. C75 (1997) 367. 